\title{
Belastung und Quellen organischer Schadstoffe im Klärschlamm und ihre Bedeutung im Zusammenhang mit dem Ausstieg aus der landwirtschaftlichen Klärschlammverwertung in der Schweiz
}

\author{
Organic contaminants in sewage sludge, their sources and their \\ importance in the context of the withdrawal from the agricultural \\ use of sewage sludge in Switzerland
}

\author{
von T. KUPPER
}

\begin{abstract}
KURZFASSUNG/SUMMARY
Klärschlamm enthält eine Vielzahl von organischen Schadstoffen und ist eine geeignete Matrix zur Beobachtung anthropogener Emissionen Polyzyklische aromatische Kohlenwasserstoffe (PAK), polychlorierte Biphenyle (PCB), Organochlorpestizide, Phthalate, UV Filter, Biozide, synthetische Duftstoffe, polybromierte Flammschutzmittel und Tenside wurden im stabilisierten Klärschlamm von über 20 Standorten (Kläranlage, Einzugsgebiet) eines Beobachtungsnetzes in der Schweiz analysiert. Die Konzentrationen der untersuchten Stoffe lagen im Bereich von wenigen $\mu \mathrm{g} / \mathrm{kg}$ TS (Trockensubstanz) bis zu einigen $\mathrm{g} / \mathrm{kg}$ TS. Die berechneten Schadstofffrachten, welche bei landwirtschaftlicher Klärschlammverwertung auf die Böden gelangen, waren bedeutend im Vergleich zu anderen Eintragspfaden.

Die spezifischen Frachten (d.h. pro angeschlossenem Einwohnerln) im Klärschlamm der drei Typen von Beobachtungsstandorten zeigten für alle Stoffe eine Hintergrundbelastung aus den privaten Haushalten auf. Die atmosphärische Deposition war für PAK, PCB, Biozide und polybromierte Flammschutzmittel die Hauptquelle. Synthetische Duftstoffe wurden fast nur von den privaten Haushalten freigesetzt. Seit 2006 ist vorwiegend aus Gründen nicht genau kalkulierbarer gesundheitlicher und ökotoxikologischer Risiken sowie des Marktrisikos die Verwendung von Klärschlamm in der Landwirtschaft verboten. Die Entsorgung mittels Verbrennung erfolgt in der Schweiz nun weitestgehend autonom.
\end{abstract}

Sewage sludge contains a multitude of organic substances and is a suitable matrix for the study of anthropogenic emissions. Polycyclic aromatic hydrcarbons (PAHs), polychlorinated biphenyls (PCBs), organochlorine pesticides, phthalates, UV filters, biocides, synthetic fragrances, polybrominated flame retardants and surfactants have been analysed in stabilised sewage sludge from more than 20 locations (water treatment works, catchment area) in a monitoring network in Switzerland. The concentrations of the analysed substances ranged between few $\mu \mathrm{g} / \mathrm{kg}$ and several $\mathrm{g} / \mathrm{kg}$ of dry matter. The calculated loads of contaminants which end up in soils where sludge is used in agriculture were substantial compared with other input pathways.

The specific loads (per connected inhabitant) in the sewage sludge from the three location types studied revealed some background contamination from private households for all analysed substances. The main source for PAHs, PCBs, biocides and polybrominated flame retardants was atmospheric deposition. Synthetic fragrances were emitted almost only by private households.

Since 2006, the use of sewage sludge in agriculture has been banned for reasons of health and ecotoxicological risks difficult to assess as well as in view of the market risk. Disposal by incineration is now largely autonomous in Switzerland.

\section{EINLEITUNG}

Verschiedene Gruppen organischer Schadstoffe wurden in jüngster Zeit in deraquatischen Umwelt nachgewiesen. Das ubiquitäre Auftreten dieser Stoffe und potenzielle Auswirkungen werden für die Umwelt als problematisch beurteilt (SCHWARZENBACH et al., 2006). Eine Vielzahl dieser Stoffe ist in der Siedlungenwässerung zu finden. Nach ihrer Verwendung gelangen die Stoffe mit dem Abwasser direkt oder via atmosphärische Deposition mit dem Abfluss von befestigten Flächen in die Kanalisation. In den Kläranlagen sorbieren lipophile Verbindungen zum größten Teil an den Schlamm. Klärschlamm enthält deshalb eine Vielzahl von umweltrelevanten Verbindungen und ist als Matrix zur Charakterisierung der Emissionen von Problemstoffen verwendbar. Für die
Erfassung der Quellen von Schadstoffen und ihrer Verteilung in der Umwelt auf der Grundlage von Klärschlamm müssen die Abwasserreinigungstechnologie und die Eigenschaften des Einzugsgebiets der Kläranlagen, insbesondere das Entwässerungssystem, die sozioökonomische Struktur sowie industrielle und gewerbliche Aktivitäten bekannt sein. Deshalb wurde im Rahmen des Projekts „Beobachtung des Stoffwechsels der Anthroposphäre im Einzugsgebiet ausgewählter Abwasserreinigungsanlagen" (SEA) ein Beobachtungsnetz bestehend aus rund 30 Untersuchungsstandorten mit drei Standortstypen (A, B, C) aufgebaut. Das Einzugsgebiet vom Typ A ist ländlich. Es weist keine Industrie bzw. nur einzelne gewerbliche Betriebe auf. Die Entwässerung erfolgt mittels Trennsystem. Der Klärschlamm an diesen
Standorten enthält fast ausschließlich Stoffe, die in den privaten Haushalten verbraucht werden. Das Einzugsgebiet von Typ B hat vergleichbare Eigenschaften, wird jedoch im Mischsystem entwässert. Zusätzlich zu den Stoffen aus den privaten Haushalten sind im Klärschlamm die im Oberflächenabfluss enthaltenen Stoffe vorhanden. Typ C umfasst eher städtische Standorte. Die Entwässerung erfolgt im Mischsystem. Aufgrund der Ansiedlung von Industrie im Einzugsgebiet sind im Klärschlamm Stoffe industrieller Herkunft zu finden. Somit können die folgenden Hauptquellen von Schadstoffen bestimmt werden: die privaten Haushalte (Typ A), die atmosphärische Deposition (Typ B) sowie Industrie und Gewerbe (Typ C). Mit dem Projekt SEA steht so ein kostengünstiges und wirksames Instrument 
zur Verfügung, um umweltrelevante Verbindungen $\mathrm{zu}$ beobachten, die Haupteintragsquellen zu bestimmen, Trends zu erkennen und Maßnahmen zur Verminderung der Einträge auszuarbeiten sowie die Wirkungs- und Erfolgskontrolle bei Maßnahmen zur Regulierung von Stoffflüssen und zum Schutz der Gewässer durchzuführen. Dies betrifft auch die landwirtschaftliche Klärschlammverwertung, da diese Stoffe durch die Ausbringung von Klärschlamm in die Böden gelangen und dadurch den Boden, die terrestrischen Ökosysteme und die Nahrungskette belasten können (vgl. SELLSTRÖM et al., 2005).

Aufgrund der Umweltrelevanz verschiedener Klassen von organischen Schadstoffen wurden im Rahmen des Projekts SEA verschiedene Studien mit folgenden Zielen durchgeführt: Bestimmen (i) der Konzentrationen, (ii) der Frachten von organischen Schadstoffen im Klärschlamm sowie ihres Eintrags bei landwirtschaftlicher Klärschlammverwertung in die Böden sowie (iii) Bezeichnung der Hauptquellen von organischen Schadstoffen. Diese Ausführungen sind in Kapitel 2 enthalten. Die Vielfalt von organischen Schadstoffen im Klärschlamm hat neben anderen Faktoren zum Ausstieg aus der landwirtschaftlichen Klärschlammverwertung in der Schweiz geführt. In Kapitel 3 werden Vorgeschichte und Gründe für diesen Entscheid sowie die Umsetzung beschrieben.

\section{BELASTUNG UND OUELLEN ORGANISCHER SCHADSTOFFE IM KLÄRSCHLAMM}

2.1 Material und Methoden 2.1.1 Untersuchte Stoffgruppen

Im Rahmen des Projekts SEA wurden die folgenden Stoffgruppen untersucht:

- Polyzyklische aromatische Kohlenwasserstoffe, 16 PAK-LeitverbindungenderEPA:Naphthalin(NAP), Acenaphthylen (ACY), Acenaphthen (ACE), Fluoren (FLU), Phenanthren (PHE), Anthracen (ANT), Fluoranthen (FLT), Pyren (PYR), Benzo(a)anthracen (BaA), Chrysen (CHR), Benzo(b)fluoranthen (BbF), Benzo(k)fluoranthen $(\mathrm{BkF})$, Benzo(a)pyren (BaP), Indeno(1,2,3-c,d)pyren (IND), Dibenzo(a,h)anthracen (DBA) und Benzo(g,h,i)perylen (BGP)

- Polychlorierte Biphenyle (PCB, Kongeneren Nr. 28, 52, 101, 118, $138,153,180$ )

- Organochlorpestizide (HCB, $\beta$ -
$\mathrm{HCH}$, Lindan, Heptachlor, Heptachlor-Epoxid (Hept.-Ep), Aldrin, Endosulfan, DDE, Dieldrin, Endrin, DDD, DDT)

- Dimethylphthalat (DMP), Diethylphthalat, (DEP), Diisobutylphthalat (DIBP), Dibutylphthalat (DBP), bis(2-Ethylhexyl)phthalat (DEHP), Dicyclohexylphthalat (DCHP), Din-octylphthalat (DnOP), Di-nnonylphthalat (DnNP), Hexyl-2ethylhexylphthalat, bis(2-Methoxyethyl)phthalat, bis(4-Methyl2-pentyl)phthalat, bis(2-Ethoxyethyl)phthalat, Diamylphthalat, Dihexylphthalat, Butylbenzylphthalat und bis(2-n-Butoxyethyl-) phthalat

- UV Filter: Methylbenzylidencampher (4-MBC), Octylmethoxycinnamat (OMC), Octocrylen (OC) und Octyltriazon (OT)

- Biozide: Carbendazim (Carb.), Diuron, Irgarol $1051^{\circ}$, Octhilinon, Permethrin (Perm.), Tributylzinn (TBT) und Triphenylzinn (TPT)

- Synthetische Duftstoffe: polyzyklische Moschus-Verbindungen (PMV) mit folgenden Einzelverbindungen: HHCB (Galaxolid), AHTN (Tonalid), ADBI (Celestolid), AHMI (Phantolid) und ATII (Traseolid) sowie der Metabolit HHCB-Lakton

- Polybromierte Flammschutzmittel: polybromierte Diphenylether, PBDE als pentaBDE (berechnet aus den folgenden Einzelkongeneren nach IUPAC: BDE28, BDE47, BDE49, BDE66, BDE85, BDE99, BDE100, BDE119, BDE138, BDE153, BDE154), octaBDE (berechnet mittels BDE183) und decaBDE (BDE209) sowie Hexabromcyclododecan (HBCD; vgl. KUPPER et al., 2007)

- Nonylphenolpolyethoxylate (NPnEO) und lineare Alkylbenzolsulfonate (LAS) mit NP, NP1EO, NP2EO bzw. C-10-LAS, C-11-LAS, C-12-LAS, C-13-LAS als untersuchte Einzelverbindungen.

\subsubsection{Beschreibung der untersuchten Stoffgruppen}

PAK sind Produkte unvollständiger Verbrennungsprozesse von Kohlenstoffwasserstoffen und entstehen z. B. bei Heizkraftwerken, bei der Müllverbrennung und in Verbrennungsmotoren. PCB wurden seit den $40 \mathrm{er}$ Jahren in großem Umfang hergestellt (weltweit produzierte Menge 1,5 Mio. Tonnen). Sie wurden insbesondere für Transformatoren und Kondensatoren, als Weichmacher für Kunststoffe, als
Hydraulikflüssigkeit, als Zusatz zu Papier, Wachsen etc. eingesetzt. Die Organochlor-Pestizide (OCP) umfassen eine Reihe hochchlorierter cycloaliphatischer Verbindungen (Aldrin, Dieldrin, HCH-Isomeren, Lindan) sowie polyhalogenierte Aromaten, von denen DDT zu den bekanntesten Vertretern gehört. PCB und die meisten der untersuchten OCP gehören zu den so genannten POPs („Persistent Organic Pollutants"). Ihre Verwendung wurde im Rahmen der POP Konvention („Stockholm Convention on Persistent Organic Pollutants") in den Industrieländern verboten. Phthalate gehören als Weichmacher für Kunststoffe zu den wichtigsten organischen Chemikalien, wobei DEHP den größten Anteil ausmacht. UV-Filter werden in Sonnenschutzmitteln eingesetzt. Sie finden zudem als Stabilisatoren in zahlreichen Kosmetikprodukten und Kunststoffen Verwendung. Biozide werden hauptsächlich zum Schutz von Fertigerzeugnissen gegen mikrobielle Schädigung zwecks Verlängerung ihrer Haltbarkeit, zur Desinfektion, zum Schutz von Oberflächen, Farben, Mauerwerk und Holz etc. eingesetzt. PMV finden in Produkten wie Waschund Reinigungsmitteln, Produkten zur Körperpflege, Parfums sowie in Raumerfrischern breite Verwendung. Polybromierte Flammschutzmittel sind synthetische Additive, welche in entflammbaren Materialien wie Kunststoffen, Textilien und Baumaterialien sowie in elektrischen und elektronischen Geräten eingesetzt werden. NPnEO sind in Industriereinigern, in Hilfsmitteln für die Textilverarbeitung und in weiteren Produkten enthalten. LAS sind wie die NPnEO oberflächenaktive Stoffe, die zur Produktion von Reinigungsmitteln verwendet werden. Die Verwendungsmengen der untersuchten Stoffgruppen liegen für Europa in der Größenordnung von $10^{3}$ t pro Jahr (für NPnEO und LAS in der Größenordnung von $10^{5} \mathrm{t}$ pro Jahr und Phthalate $10^{6} \mathrm{t}$ pro Jahr).

\subsubsection{Probenahme und Analyse- methoden und Eigenschaften der Kläranlagen}

Für die Laboranalysen wurde der aerob oder anaerob stabilisierte Klärschlamm von Kläranlagen der Standorte des Typs A, B und C verwendet. Die Entnahme der Proben erfolgte aus dem Stapelbehälter. Die Beprobungskampagnen fanden im Jahr 1998 für die Untersuchung der PAK, PCB, OCP und Phthalate statt. Die Proben für die Untersuchung von UV-Filtern, Biozi- 
den und PMV wurden 2001 sowie im Jahr 2003 für die Analyse von PBDE und $\mathrm{HBCD}$ entnommen. Die Probenahme für die Analysen der NPnEO und LAS erfolgte in den Jahren 2000, 2001 und 2004. Probenahme, Analysemethoden und die Eigenschaften der Kläranlagen sind in BERSET und HOLZER (1999, 2001), KUPPER et al. (2004; 2007), PLAGELLAT et al. (2004; 2006) und WETTSTEIN (2004) beschrieben.

\subsubsection{Berechnung der spezifischen Fracht}

Die Konzentration eines Stoffs im Klärschlamm ist nicht in jedem Fall geeignet, um die Immissionssituation $\mathrm{zu}$ beschreiben. So kann etwa eine hohe Klärschlammproduktion z.B. infolge eines hohen Anteils von Abwasser aus der Lebensmittelindustrie zur Verdünnung eines untersuchten Stoffs führen (KUPPER et al., 2004). Die normalisierte Fracht eines Stoffs wird von solchen Effekten nicht beeinflusst. In der vorliegenden Studie wird die spezifische Fracht bestimmt. Sie ist wie folgt definiert:

$$
\mathrm{F}_{\mathrm{sp}(i)}=\mathrm{K}_{\mathrm{Ks}(i)} \times \mathrm{P}_{\mathrm{Ks}} \times \mathrm{cap}^{-1}
$$

$\mathrm{F}_{\text {sp }(i)}$ : spezifische Fracht des Stoffs $i$; Fracht im Klärschlamm pro angeschlossenem/angeschlossener EinwohnerIn und Jahr (mg cap $\left.{ }^{-1} \mathrm{a}^{-1}\right)$

$\mathrm{K}_{\mathrm{KS}(i)}$ : Konzentration des Stoffs $i$ im aerob oder anaerob stabilisierten Klärschlamm ( $\mu \mathrm{g} / \mathrm{kg} \mathrm{TS}$; Trockensubstanz)

$P_{K s}$ : Produktion von aerob oder anaerob stabilisiertem Klärschlamm (t TS/a)

cap: Anzahl der an die Kläranlage angeschlossenen EinwohnerInnen

\subsection{Resultate und Diskussion 2.2.1 Konzentrationen der untersuchten Stoffgruppen im Klärschlamm}

Die Analyseresultate der untersuchten Stoffe sind in Tabelle 1a-d aufgeführt. Die Summe der 16 PAK lag im Mittel bei $7160 \mu \mathrm{g} / \mathrm{kg}$ TS. Die 3-Ring-Verbindungen machten mit rund $40 \%$ den größten Anteil der Einzelverbindungen aus.

Die Summe der 7 PCB lagen bei durchschnittlich 93,5 $\mu \mathrm{g} / \mathrm{kg}$ TS, wobei die höher chlorierten Kongeneren tendenziell stärker vertreten waren. HCB, Lindan, Aldrin, Heptachlor-Epoxid, Endosulfan, DDE, DDD, DDT wurden in sämtlichen oder zumindest in der Mehrzahl der Proben mit mittleren Gehalten zwischen $2,1 \mu \mathrm{g} / \mathrm{kg}$ TS (HCB) und $72 \mu \mathrm{g} / \mathrm{kg}$ TS (Hepta-
chlor-Epoxid) nachgewiesen. Dieldrin wurde in $5, \beta-\mathrm{HCH}$ in 3 , Heptachlor in 2 und Endrin in einer Probe nachgewiesen. Die Gehalte lagen meist im niedrigen ppb-Bereich. Das Auftreten von $\mathrm{PCB}$ und OCP kann trotz Verwendungsverboten durch ihre Persistenz erklärt werden.

Die Phthalate lagen in Konzentrationen zwischen $4,1 \mu \mathrm{g} / \mathrm{kg}$ TS (DMP) und $51,2 \mathrm{mg} / \mathrm{kg}$ TS (DEHP) vor. Hexyl-2-ethylhexylphthalat erreichte in 4 Proben Konzentrationen zwischen 10 und $67 \mu \mathrm{g} / \mathrm{kg}$ TS. Bis(2-Methoxyethyl)phthalat, bis(4Methyl-2-pentyl)phthalat, bis(2-Ethoxyethyl)phthalat, Diamylphthalat, Dihexylphthalat, Butylbenzylphthalat und bis(2-n-Butoxyethyl)phthalat wurden nicht gefunden. Die verhältnismäßig hohen Gehalte (insbesondere DEHP) widerspiegeln die weite Verbreitung dieser Stoffe in der Anthroposphäre.

Die mittleren Gehalte der UV-Filter 4-MBC, OC und OT sowie der polyzyklischen Moschus-Verbindungen HHCB, AHTN und HHCB-Lakton lagen in der Größenordnung von $10^{3} \mu \mathrm{g} / \mathrm{kg}$ TS. Die Konzentrationen des UV-Filters OMC, der Biozide Carbendazim, Diuron, Permethrin, TBT und TPT sowie der Duftstoffe ADBI, AHDI, ATII, der PBDE sowie HBCD betrugen rund $10-100 \mu \mathrm{g} / \mathrm{kg}$ TS Sämtliche Verbindungen kamen in allen Proben vor mit Ausnahme von TPT und Irgarol $1051^{\circ}$, welche in 11 bzw. 7 von 24 Proben gefunden wurden. Einzig Octhilinon konnte nicht nachgewiesen werden.

Die Proben zur Analyse von UVFiltern, Bioziden und PMV wurden im Winter sowie im Frühjahr/Sommer entnommen. Die Einzeldaten der beiden Beprobungskampagnen unterschieden sich um bis zu Faktor 5. Die mittleren Gehalte der UV-Filter waren im Sommer tendenziell höher, aber nur OT zeigte einen signifikanten Unterschied $(p<0,05)$. Dies dürfte dadurch zu erklären sein, dass diese Verbindung ausschließlich in Sonnenschutzmitteln enthalten ist, welche im Sommer in größeren Mengen verbraucht werden. Die durchschnittlichen Konzentrationen der Biozide waren im Winter tendenziell höher als im Frühjahr. Die Variabilität der PMV war gering (größtenteils $<25 \%$ ).

Die mittleren Werte für die Summe der NPnEO lagen im Jahr 2004 bei $40 \mathrm{mg} / \mathrm{kg}$ TS mit NP als wichtigster Einzelverbindung. Die LAS lagen bei rund $2,5 \mathrm{~g} / \mathrm{kg}$ TS. Von den NPnEO und den LAS liegen Analyseresultate von 2000, 2001 und 2004 vor. Wie
Abb. 1 zeigt, ist unter Berücksichtigung der großen Standardabweichungen kein Trend bezüglich Klärschlammbelastung erkennbar. Im Jahr 1986 wurde der Einsatz von Octyl- und Nonylphenolpolyethoxylaten in Textilwaschmitteln gesetzlich verboten. Dies zeigte sich in stark abnehmenden Gehalten im Klärschlamm und im Abwasser in den 1990er Jahren (WETTSTEIN, 2004). Seither wurde keine weitere Reduktion erreicht. Die LAS werden weiterhin in großen Mengen eingesetzt, was sich in den hohen und konstanten spezifischen Frachten widerspiegelt.

Zusammenfassend lässt sich sagen, dass PAK, DEHP, NPnEO (mg/ $\mathrm{kg}$ TS) und LAS ( $\mathrm{g} / \mathrm{kg}$ TS) in relativ hohen Konzentrationen vorliegen. Die klassischen persistenten organischen Schadstoffgruppen $\mathrm{PCB}$ und OCP weisen niedrige Konzentrationen auf. Die Belastung mit solchen Stoffen ist vermutlich abnehmend. Neuere Stoffklassen, die teilweise POP-ähnliche Eigenschaften (PMV, UV-Filter, PBDE, HBCD), aber im Vergleich zu den klassischen POP ein niedrigeres Risikopotenzial aufweisen, liegen in deutlich höheren und in der Tendenz vermutlich zunehmenden Konzentrationen vor. Das Vorkommen von Stoffen mit hoher Umweltrelevanz nimmt im Klärschlamm ab. Gleichzeitig vergrößert sich die Anzahl neuerer Stoffe.

\subsubsection{Eintrag von organischen Schad- stoffen in die landwirtschaftlichen Böden}

Die Bedeutung des Eintrags von organischen Schadstoffen in die Böden infolge der Verwendung von Klärschlamm als Dünger kann abgeschätzt werden, indem die Frachten mit anderen Quellen (Kompost, Gärgut, Presswasser, Hofdünger, atmosphärische Deposition) verglichen werden. Dabei werden zwei Ansätze verfolgt: Berechnung (i) der Gesamtfracht, welche in die landwirtschaftliche Nutzfläche der Schweiz eingetragen wird, und (ii) der flächenspezifischen Fracht (Fracht pro Hektare landwirtschaftliche Nutzfläche bei üblichen Ausbringungsmengen (normiert auf $70 \mathrm{~kg} \mathrm{P}_{2} \mathrm{O}_{5}$; vgl. BRÄNDLI et al., 2007a, b). Im Folgenden werden beispielhaft die Resultate von PAK und DEHP diskutiert.

Die atmosphärische Deposition war die wichtigste Quelle von PAK für die gesamte landwirtschaftliche Nutzfläche der Schweiz (ca. $1700 \mathrm{~kg}$ pro Jahr; Abb. 2). Die niedrige Fracht von rund $290 \mathrm{~kg}$ pro Jahr im Klärschlamm 
Tabelle 1a. Konzentrationen von PAK, PCB und Organchlorpestizide im Klärschlamm in $\mu g / k g$ TS.

\begin{tabular}{|c|c|c|c|c|c|c|c|}
\hline Stoff & Mittelwert & Min. & $10 \%$ Perz. & Median & $90 \%$ Perz. & Max. & Stabw. \\
\hline \multicolumn{8}{|c|}{ PAK in $\mu \mathrm{g} / \mathrm{kg}$ TS (12 Standorte; $\mathrm{n}=12$ ) Quelle: BERSET und HOLZER (1999) } \\
\hline NAP & 142 & 73 & 73 & 99 & 209 & 478 & 114 \\
\hline $\mathrm{ACY}$ & 15,2 & 3,6 & 4,3 & 17,4 & 22,1 & 23,8 & 7,2 \\
\hline ACE & 152 & 34 & 37 & 106 & 275 & 506 & 134 \\
\hline FLU & 185 & 46 & 48 & 131 & 385 & 633 & 170 \\
\hline PHE & 799 & 152 & 198 & 769 & 1452 & 1720 & 493 \\
\hline ANT & 79 & 12 & 12 & 73 & 132 & 220 & 58 \\
\hline FLU & 1197 & 171 & 429 & 1087 & 1763 & 3767 & 960 \\
\hline PYR & 974 & 153 & 305 & 927 & 1397 & 3260 & 837 \\
\hline $\mathrm{BaA}$ & 415 & 76 & 81 & 393 & 569 & 1701 & 446 \\
\hline CHR & 592 & 86 & 104 & 539 & 864 & 2186 & 581 \\
\hline $\mathrm{BbF}$ & 758 & 119 & 120 & 660 & 1061 & 2918 & 765 \\
\hline $\mathrm{BkF}$ & 327 & 56 & 58 & 289 & 438 & 1267 & 329 \\
\hline $\mathrm{BaP}$ & 511 & 93 & 94 & 449 & 697 & 2093 & 546 \\
\hline IND & 470 & 76 & 76 & 422 & 658 & 1827 & 477 \\
\hline DBA & 113 & 20 & 21 & 107 & 159 & 422 & 109 \\
\hline BGP & 432 & 76 & 76 & 423 & 614 & 1542 & 402 \\
\hline Summe & 7160 & 1726 & 1760 & 6688 & 10507 & 22641 & 5661 \\
\hline \multicolumn{8}{|c|}{ PCB in $\mu \mathrm{g} / \mathrm{kg}$ TS (12 Standorte; $\mathrm{n}=12$ ) Quelle: BERSET und HOLZER (1999) } \\
\hline РCB28 & 4,6 & 1,4 & 1,9 & 3,9 & 9,0 & 9,3 & 2,8 \\
\hline PCB52 & 10,0 & 3,8 & 4,3 & 7,7 & 16,1 & 27,2 & 6,5 \\
\hline PCB101 & 16,6 & 6,8 & 9,0 & 15,4 & 23,7 & 30,7 & 7,2 \\
\hline PCB118 & 14,1 & 5,8 & 7,1 & 13,7 & 20,8 & 28,1 & 6,7 \\
\hline PCB138 & 20,8 & 8,9 & 9,8 & 20,9 & 30,5 & 30,8 & 7,9 \\
\hline PCB153 & 16,8 & 6,3 & 7,6 & 16,5 & 25,9 & 28,6 & 7,5 \\
\hline PCB180 & 10,6 & 4,3 & 5,0 & 10,4 & 14,9 & 15,9 & 4,0 \\
\hline Summe & 93,5 & 37,8 & 45,1 & 94,0 & 146 & 152 & 37,8 \\
\hline \multicolumn{8}{|c|}{ Organchlorpestizide in $\mu \mathrm{g} / \mathrm{kg}$ TS (11 Standorte; $\mathrm{n}=11$ ) Quelle: BERSET und HOLZER (1999) } \\
\hline $\mathrm{HCB}$ & 2,1 & n.n. & n.n. & 0,9 & 2,7 & 13,1 & 3,8 \\
\hline Lindan & 17,2 & n.n. & n.n. & 7,1 & 34,4 & 57,0 & 18,4 \\
\hline Aldrin & 8,6 & n.n. & n.n. & 6,4 & 14,6 & 28,9 & 8,0 \\
\hline Hept.-Ep & 72,0 & n.n. & n.n. & 85,6 & 142 & 165 & 62,9 \\
\hline Endosulfan & 10,4 & n.n. & n.n. & 12,4 & 20,9 & 23,7 & 9,2 \\
\hline $\mathrm{DDE}$ & 54,0 & n.n. & n.n. & 55,9 & 80,1 & 97,2 & 26,6 \\
\hline DDD & 25,1 & n.n. & n.n. & 24,0 & 42,1 & 47,9 & 14,3 \\
\hline DDT & 11,0 & n.n. & 7,5 & 10,9 & 15,5 & 16,7 & 4,7 \\
\hline
\end{tabular}

ist durch die abnehmende Verwertung in der Landwirtschaft im Zusammenhang mit dem Ausbringungsverbot ab Herbst $2006 \mathrm{zu}$ erklären (für 2002: $21 \%$ der gesamten Menge). Die gesamte Fracht im Klärschlamm würde rund $1400 \mathrm{~kg}$ pro Jahr betragen und liegt damit deutlich höher als die Fracht in Kompost und Gärgut (570 kg pro Jahr) sowie Hofdünger (360 kg pro Jahr; Brändli et al., 2007a). Klärschlamm verursacht dagegen eine niedrigere flächenspezifische Fracht als etwa Kompost. Dies ist auf den hohen P-Gehalt von Klärschlamm und die nicht vernachlässigbaren PAK-Gehalte in Kompost sowie verhältnismäßig hohe Ausbringungsmengen dieses Recyclingdüngers zurückzuführen. Auch bei DEHP sind die Frachten im Klärschlamm bedeutend, wobei hier auch die hohe flächenspezifische Fracht auffällt. Wenn auch der Eintrag eines Schadstoffs in den Boden allein noch nichts über die Gefährdung für den Boden aussagt, weist dies doch darauf hin, dass ein gewisses Risikopotenzial besteht. Dieses steigt bei zunehmenden Frachten an.

\subsubsection{Spezifische Frachten der} untersuchten Stoffgruppen im Klärschlamm - Bestimmung der Quellen

Die spezifischen Frachten von PAK, PCB, UV-Filtern, Permethrin, 
Tabelle 1b. Konzentrationen von Phthalaten im Klärschlamm in $\mu g / k g$ TS

\begin{tabular}{|c|c|c|c|c|c|c|c|}
\hline Stoff & Mittelwert & Min. & $10 \%$ Perz. & Median & $90 \%$ Perz. & Max. & Stabw. \\
\hline \multicolumn{8}{|c|}{ Phthalate (12 Standorte; $n=12$ ) Quelle: BERSET und HOLZER (2001) } \\
\hline DMP & 4,1 & n. n. & n. n. & n. n. & 11,0 & 27,0 & 8,4 \\
\hline DEP & 47 & 0 & 0 & 30 & 136 & 145 & 56 \\
\hline DIBP & 151 & 80 & 97 & 116 & 296 & 346 & 86 \\
\hline DBP & 512 & 193 & 230 & 412 & 983 & 1257 & 326 \\
\hline DEHP & 51212 & 21055 & 23923 & 33361 & 110579 & 113864 & 36646 \\
\hline DCHP & 291 & n. n. & n. n. & 100 & 907 & 1047 & 381 \\
\hline DnOP & 137 & n. n. & n. n. & n. n. & 342 & 629 & 200 \\
\hline DnNP & 404 & n. n. & n. n. & 316 & 661 & 1583 & 450 \\
\hline
\end{tabular}

Tabelle 1c. Konzentrationen von UV Filtern, Bioziden, polyzyklischen Moschus-Verbindungen (PMV)und polybromierten Flammschutzmitteln (PBDE, HBCD) im Klärschlamm in $\mu \mathrm{g} / \mathrm{kg}$ TS

\begin{tabular}{|c|c|c|c|c|c|c|c|}
\hline Stoff & Mittelwert & Min. & $10 \%$ Perz. & Median & $90 \%$ Perz. & Max. & Stabw. \\
\hline \multicolumn{8}{|c|}{ UV Filter in $\mu \mathrm{g} / \mathrm{kg}$ TS (12 Standorte; $\mathrm{n}=24$ ) Quelle: PLAGELLAT et al. (2006) } \\
\hline $4-\mathrm{MBC}$ & 1777 & 148 & 252 & 1573 & 3395 & 4976 & 1349 \\
\hline OMC & 110 & 12 & 30 & 99 & 197 & 392 & 85 \\
\hline $\mathrm{OC}$ & 4834 & 322 & 874 & 3269 & 10308 & 18738 & 4520 \\
\hline OT & 5517 & 685 & 1308 & 3463 & 11398 & 27736 & 5767 \\
\hline \multicolumn{8}{|c|}{ Biozide in $\mu \mathrm{g} / \mathrm{kg}$ TS (12 Standorte; $\mathrm{n}=24$ ) Quelle: PLAGELLAT et al. (2004) } \\
\hline Carb. & 6,8 & 1,6 & 2,0 & 5,1 & 17 & 21 & 6,0 \\
\hline Diuron & 9,5 & 0,6 & 1,2 & 5,9 & 17 & 44 & 9,5 \\
\hline Irgarol & 10 & 1,5 & 1,6 & 5,9 & 24 & 30 & 11 \\
\hline Perm. & 98 & 10 & 37 & 87 & 180 & 216 & 59 \\
\hline TBT+ & 148 & 19 & 24 & 121 & 285 & 649 & 152 \\
\hline TPT+ & 44 & 4,3 & 5,4 & 18 & 40 & 283 & 80 \\
\hline \multicolumn{8}{|c|}{ PMV in $\mu \mathrm{g} / \mathrm{kg}$ TS (16 Standorte; $\mathbf{n}=21$ ) Quelle: KUPPER et al. (2004) } \\
\hline НHCB & 20336 & 11571 & 12489 & 19679 & 28875 & 31397 & 6357 \\
\hline AHTN & 7262 & 3398 & 4360 & 6984 & 10890 & 11203 & 2560 \\
\hline ADBI & 427 & 139 & 184 & 316 & 766 & 1087 & 275 \\
\hline AHMI & 811 & 407 & 525 & 692 & 1175 & 1663 & 328 \\
\hline ATII & 672 & 256 & 400 & 681 & 967 & 1010 & 231 \\
\hline HHCB-L. & 1781 & 754 & 846 & 1610 & 3235 & 3319 & 874 \\
\hline \multicolumn{8}{|c|}{ Polybromierte Flammschutzmittel in $\mu \mathrm{g} / \mathrm{kg}$ TS (16 Standorte; $n=19$ ) Quelle: KUPPER et al. (2007) } \\
\hline PentaBDE & 95 & 49 & 54 & 81 & 157 & 248 & 55 \\
\hline OctaBDE & 17 & 8 & 10 & 17 & 23 & 24 & 5 \\
\hline DecaBDE & 310 & 138 & 149 & 282 & 494 & 617 & 140 \\
\hline $\mathrm{HBCD}$ & 149 & 39 & 66 & 123 & 297 & 597 & 91 \\
\hline
\end{tabular}

polyzyklischen Moschus-Verbindungen und HBCD im Klärschlamm sind in Abb. 3 dargestellt. PAK, UV Filter (OC) und PMV (HHCB) weisen ähnlich hohe Frachten auf. Diese liegen um eine bis zwei Zehnerpotenzen höher im Vergleich zu PCB, Permethrin und HBCD. Die relativ niedrigen Mengen von PCB dürften auf das Verwendungsverbot zurückzuführen sein. Bei Stoffen wie OC,
HHCB und HBCD, die vergleichbare Verbrauchsmengen aufweisen und aufgrund der hohen Octanol-Wasser Verteilungskoeffizienten $\left(\log K_{o w}>5\right)$ überwiegend an den Schlamm sorbieren (KUPPER et al., 2006), wären ähnlich hohe Frachten zu erwarten. Die beobachteten Unterschiede dürften hauptsächlich darauf zurückzuführen sein, dass UV-Filter und Duftstoffe in vielen Produkten vorkommen, welche nach dem Gebrauch direkt ins Abwasser gelangen (z.B. Produkte zur Körperpflege, Kosmetika). Bei den Bioziden und den HBCD überwiegen vermutlich indirekte Einträge, sodass nur ein Teil der gesamten emittierten Stoffmenge überhaupt in die Abwasserreinigungsanlagen gelangt.

Bei sämtlichen Stoffen besteht an den Standorten des Typs A eine Grundbelastung, welche auf das häus- 
Tabelle 1d. Konzentrationen von Nonylphenolpolyethoxylate (NPnEO) und linearen Alkylbenzolsulfonaten (LAS) im Klärschlamm in mg/kg TS (2004)

\begin{tabular}{|c|c|c|c|c|c|c|c|}
\hline Stoff & Mittelwert & Min. & $10 \%$ Perz. & Median & 90 \% Perz. & Max. & Stabw. \\
\hline \multicolumn{8}{|c|}{ NPnEO in mg/kg TS (21 Standorte; $\mathbf{n}=21$ ) Quelle: unveröffentlichte Daten der Eawag } \\
\hline NP & 30,2 & 2,9 & 4,6 & 25,0 & 68,0 & 126 & 30,4 \\
\hline NP1EO & 6,8 & 0,7 & 1,3 & 3,3 & 15,0 & 52,0 & 11,3 \\
\hline NP2EO & 3,3 & 0,9 & 1,4 & 2,6 & 5,6 & 8,4 & 2,0 \\
\hline Summe & 39,4 & 5,6 & 9,4 & 32,5 & 74,2 & 183 & 40,6 \\
\hline \multicolumn{8}{|c|}{ LAS in mg/kg TS (21 Standorte; $\mathbf{n}=21$ ) Quelle: unveröffentlichte Daten der Eawag } \\
\hline C-10-LAS & 96 & 2 & 12 & 80 & 180 & 280 & 73 \\
\hline C-11-LAS & 542 & 6 & 13 & 590 & 1170 & 1390 & 494 \\
\hline C-12-LAS & 949 & 20 & 25 & 960 & 2200 & 2500 & 880 \\
\hline C-13-LAS & 905 & 26 & 28 & 890 & 2090 & 2190 & 815 \\
\hline Summe & 2492 & 56 & 90 & 2520 & 5721 & 5960 & 2250 \\
\hline
\end{tabular}

liche Schmutzwasser zurückzuführen ist (Abb. 3). Für PAK, PCB, UV-Filter, Biozide und HBCD ist eine erhöhte spezifische Fracht an den Standorten des Typs B im Vergleich zu den AStandorten zu beobachten. Dies bedeutet, dass diese Stoffe an den Standorten des Typs B überwiegend mit dem Regenwasser eingetragen werden. Bei HBCD ist dies dadurch zu erklären, dass dieser Stoff in Kunststoffen nicht kovalent gebunden ist und deshalb durch Verflüchtigung freigesetzt wird. Anschließend erfolgt die Ablagerung auf befestigte Flächen und der Transport in die Kläranlagen via Oberflächenabfluss. Dies trifft vermutlich auch für UV-Filter wie OC zu, die als Stabilisatoren in Plastik eingesetzt werden. Biozide, die in Produkten zur Oberflächenbehandlung oder in Farben enthalten sind, können ausgewa- schen werden und mit dem Oberflächenabfluss ebenfalls in die Kläranlagen gelangen. Weitere Einträge an den Standorten des Typs C erfolgen durch Industrie und Gewerbe.

Die Höhe der spezifischen Frachten der PMV im Klärschlamm stimmen gut mit dem Verbrauchsmuster ( HHCB > AHTN > übrige PMV; KUPPER et al., 2004) überein. Die Höhe der spezifischen Frachten der UV-Filter ist jedoch nicht proportional zum Verbrauch. Der Stoff mit der höchsten Verbrauchsmenge (OMC) wird in der Kläranlage zu einem großen Teil abgebaut (KUPPER et al., 2006). Die Abbaubarkeitwährend Abwasserreinigung und Schlammbehandlung ist auch für die anderen Stoffe zu berücksichtigen.

Die spezifischen Frachten von NPnEO und LAS weisen bei den
Standorten des Typs A wesentlich niedrigere spezifische Frachten auf. Dies kann durch die Abbaubarkeit von NPnEO und LAS unter aeroben Bedingungen erklärt werden. Die Kläranlagen der Standorte des Typs A reinigen das Abwasser mittels Langzeitbelüftung (Schlammalter von ca. 20 Tagen). Damit sind die am Schlamm sorbierten Stoffe im Vergleich zu den Anlagen an den Standorten des Typs $B$ und $C$ über eine längere Dauer aeroben Bedingungen ausgesetzt. Somit werden NPnEO und LAS in der Abwassereinigung der Standorte des Typs A besser abgebaut, was zu den vergleichsweise niedrigeren Frachten führt. Wenn Klärschlamm als Matrix zur Umweltbeobachtung verwendet werden soll, müssen daher die Stoffeigenschaften (insbesondere Abbaubarkeit) der Stoffe berücksichtigt werden,

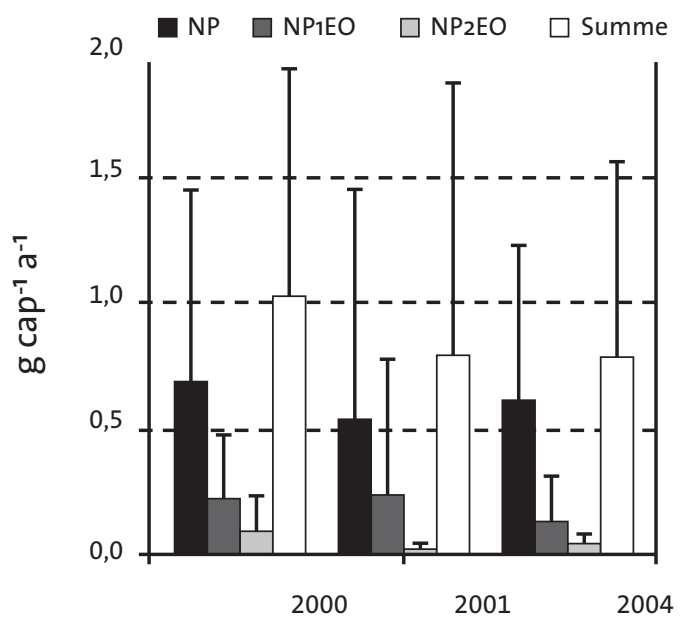

LAS

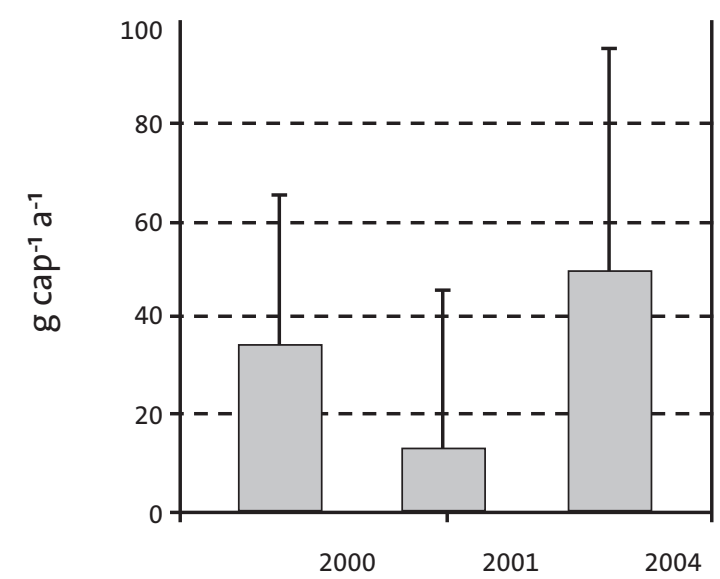

Abb. 1. Zeitreihe von NPnEO und LAS (Summe C-10-LAS bis C-13-LAS) von 2000, 2001, 2004 im Klärschlamm (spezifische Frachten: Mittelwert, Standardabweichung in $\mathrm{mg} \mathrm{cap}^{-1} \mathrm{a}^{-1}$; Quelle: unveröffentlichte Daten der Eawag, Dübendorf). 

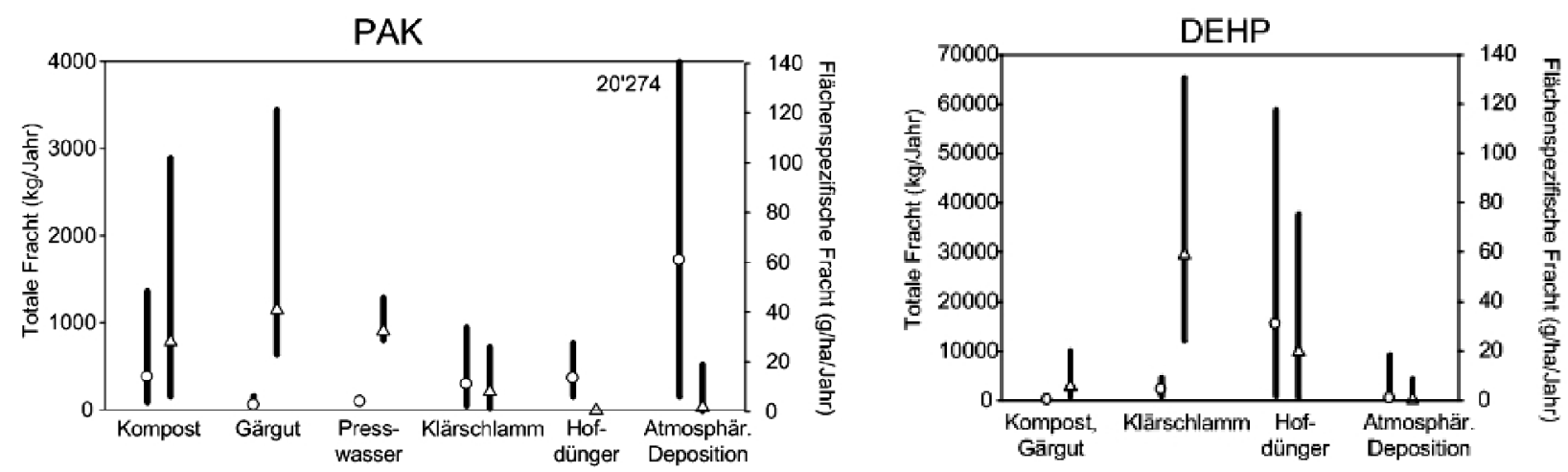

Abb. 2. Frachten pro Jahr von PAK und DEHP aus der Anwendung von Kompost, Gärgut, Presswasser, Klärschlamm, Hofdünger sowie atmosphärische Deposition. „O" entspricht der mittleren totalen Fracht in die landwirtschaftliche Nutzfläche der Schweiz in kg/Jahr und " $\Delta$ " der mittleren flächenspezifischen Fracht (Fracht pro Hektare landwirtschaftlicher Nutzfläche bei üblichen Ausbringungsmengen, normiert auf $70 \mathrm{~kg} \mathrm{P} \mathrm{P}_{5}$ ) g/ha/Jahr. Die Säulen zeigen den Bereich (Minimum und Maximum) an (Quelle: Brändli et al., 2007a,b).

um die richtigen Schlüsse im Hinblick auf ihren Eintrag in die Umwelt zu ziehen. Da nicht zu erwarten ist, dass das Verhalten eines Stoffs in einer be- stimmten Abwasserreinigungsanlage über die Zeit systematischen Veränderungen unterliegt, kann ein fest etabliertes Beobachtungsnetz dennoch zur
Langzeitbeobachtung der Emissionen von Schadstoffen verwendet werden.
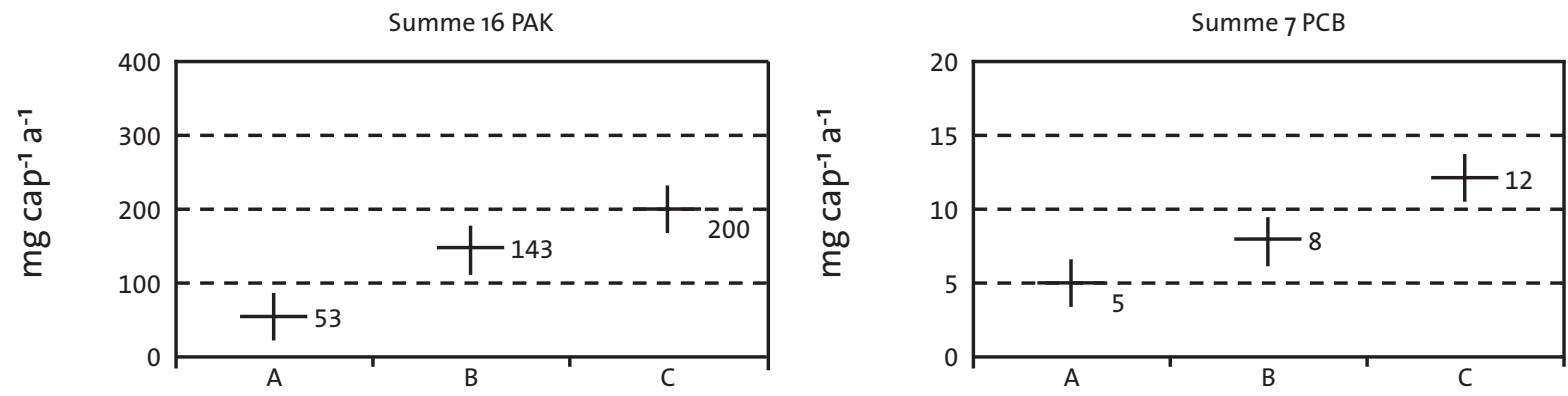

OC
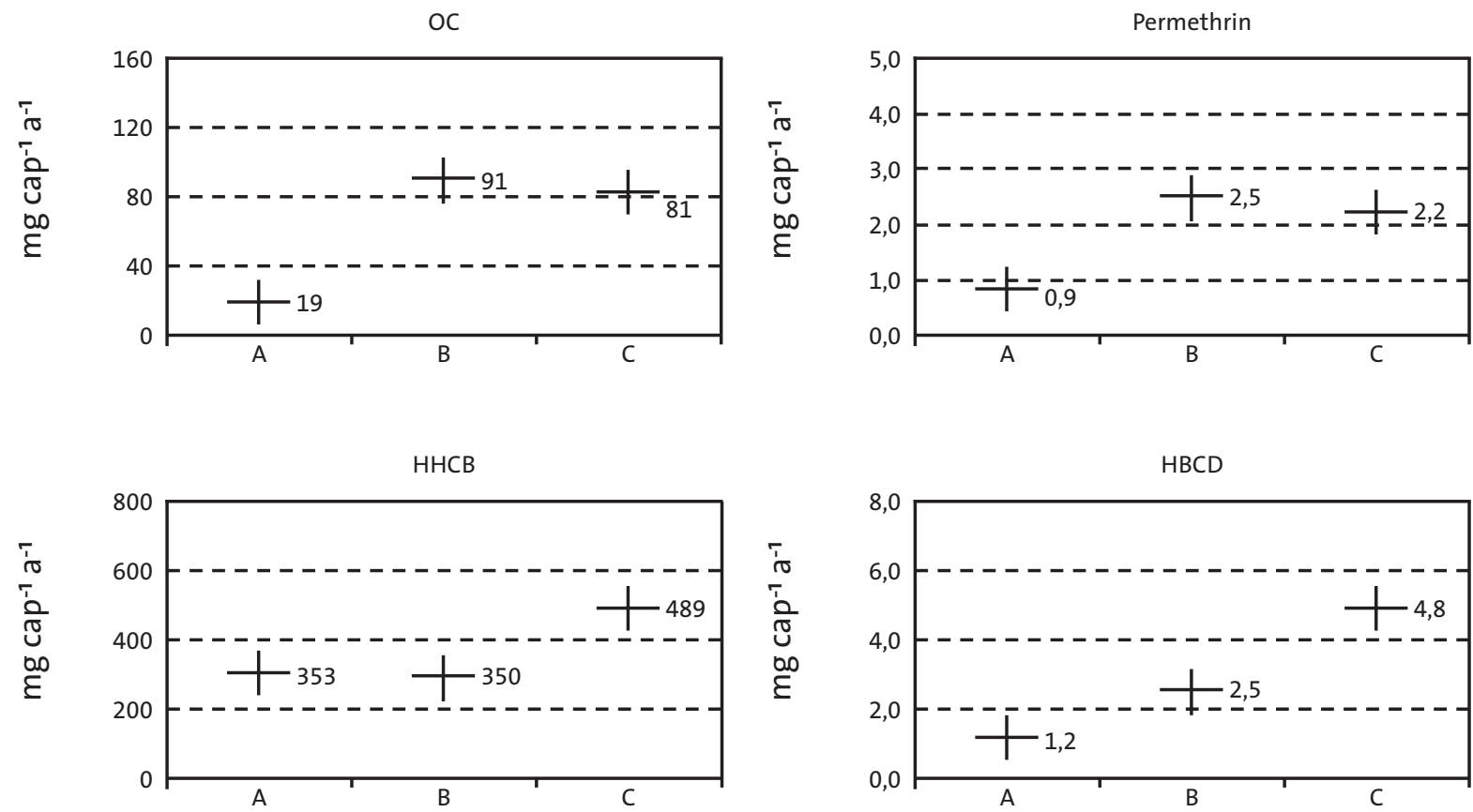

Abb. 3. Spezifische Frachten (Minimum, Mittelwert, Maximum) von PAK (Summe der 16 PAK-Leitverbindungen der EPA), PCB (Summe der Kongeneren Nr. 28 , $52,101,118,138,153,180$ ) OT (UV Filter), Permethrin (Biozid), HHCB (polyzyklische Moschus-Verbindung) und HBCD (polybromiertes Flammschutzmittel) in $\mathrm{mg} \mathrm{cap}^{-1} \mathrm{a}^{-1}$. 


\section{AUSSTIEG AUS DER LANDWIRT- SCHAFTLICHEN KLÄRSCHLAMM- VERWERTUNG IN DER SCHWEIZ}

\subsection{Vorgeschichte}

In der Schweiz wurde die landwirtschaftliche Klärschlammverwertung in der Vergangenheit verschiedentlich kontrovers diskutiert. Die Diskussion um mögliche Gesundheits- und Umweltrisiken bestand schon bei Inkrafttreten der ehemaligen Klärschlammverordnung von 1981 und bei Inkrafttreten der vorsorglichen, verschärften Vorschriften von 1992 über Dünger (Anhang 4.5 Stoffverordnung). Vor allem aufgrund wirtschaftlicher Überlegungen und dem Willen, Stoffkreisläufe zu schließen (insbesondere Rezyklierung von Phosphor), begleitet von Maßnahmen zur Verbesserung der Qualität (Hygiene, Schwermetallgehalte) und der Anwendung (Düngungsberatung, konsequente Verwendungsmengen gemäß Nährstoffbedarf der angebauten Kultur), ließ sich die Verwertung von Klärschlamm als Dünger in der Landwirtschaft beibehalten.

Im Laufe des Jahres 2000 wurden verschiedene politische Vorstöße sowie Anfragen von Verbänden und Organisationen zum Thema Abfalldünger, insbesondere Klärschlamm, lanciert. Hintergrund dazu waren Fragen zu Lebensmittelsicherheit, Gesundheit sowie Umweltauswirkungen. Dabei spielten auch die organischen Schadstoffe eine Rolle. Im Januar 2001 erreichte die Diskussion im Zusammenhang mit BSE einen neuen Höhepunkt, als Befürchtungen aufkamen, dass durch die Ausbringung von Klärschlamm von Abwasserreinigungsanlagen mit Schlachtbetrieben im Einzugsgebiet zusätzliches Risikomaterial in die Nahrungskette gelangen könnte.

\subsection{Risikoabschätzung zur landwirtschaftlichen Abfalldüngerverwertung}

Vor diesem Hintergrund hat das Bundesamt für Landwirtschaft (BLW) eine Risikoanalyse der Verwendung von Abfalldüngern in der Landwirtschaft in Auftrag gegeben. Die Resultate dieser Studie (HERTER et al., 2003) können wie folgt zusammengefasst werden: Das Verhältnis Risiko gegenüber Nutzwirkung nimmt in folgender Reihenfolge zu: Mineraldünger $<$ Abfälle aus der Nahrungsmittelindustrie < Kompost $<$ Hofdünger $<$ Abfälle aus der Holzverarbeitung < Holzasche < Klärschlamm. Abfalldünger können einen wesentlichen Beitrag zur Scho- nung der mineralischen Nährstoffreserven leisten. Ihre Beseitigung ist aus dieser Sicht nicht nachhaltig. Andererseits sind sie im Allgemeinen stärker mit Schadstoffen belastet als Hof- und Mineraldünger. Durch den Verzicht auf die landwirtschaftliche Abfalldüngerverwertung kann das Problem des Eintrags von Schadstoffen in Böden nicht gelöst werden. Dies ist nur durch eine Begrenzung des Eintrags an der Quelle möglich, das heißt durch Verzicht oder Einschränkung der Verwendung von problematischen Stoffen. Die aktuelle Praxis der Klärschlammverwendung in der Landwirtschaft sollte nach dem aktuellen Kenntnisstand kurzfristig nicht zu einer Schädigung von Mensch und Umwelt führen. Verschiedene über das Abwasser entsorgte umweltrelevante Stoffe aus privaten Haushalten und der Industrie gelangen aber in den Klärschlamm. Eine Begrenzung des Eintrags an der Quelle erscheint kurz- bis mittelfristig nicht machbar. Aus dieser Sicht stellt sich die Frage, ob das gegenwärtige Entwässerungssystem zur Gewinnung eines Stoffs von Düngerqualität geeignet ist. Ökobilanzen zur Klärschlammentsorgung zeigten (allerdings ohne Berücksichtigung der Endlichkeit der mineralischen P-Reserven und der organischen Schadstoffe) weder für die landwirtschaftliche Verwertung noch für die Verbrennung klare Vorteile, sodass eine Beibehaltung der landwirtschaftlichen Klärschlammverwertung in dieser Hinsicht nicht als zwingend erscheint.

\subsection{Umsetzung des Ausstiegs}

Im Jahr 2001 war die Haltung der vier Bundesämter - nebst dem Bundesamt für Landwirtschaft (BLW) das Bundesamt für Umwelt (Bafu), das Bundesamt für Gesundheit (BAG) und das Bundesamt für Veterinärwesen (BVET) -, teils gestützt auf die Resultate dieser Studie, wie folgt: (i) Ein sofortiger genereller Ausstieg aus der Verwendung von Abfalldüngern in der Landwirtschaft ist nicht notwendig. (ii) Mittelfristig (bis zum 30. September 2006 mit der Möglichkeit einer Verlängerung um höchstens 2 Jahre) wird - vorwiegend aus Gründen nicht genau kalkulierbarer gesundheitlicher und ökotoxikologischer Risiken sowie des Marktrisikos - der Ausstieg aus der Verwendung von Klärschlamm angepeilt. (iii) Das Risikomanagement bei Recyclingdüngern (Kompost, andere Stoffe biogener Herkunft) ist so rasch wie möglich zu verbessern.

Von Bedeutung ist in diesem $\mathrm{Zu}$ - sammenhang, dass Klärschlamm als Beurteilungsgrundlage für die Überprüfung bzw. Verschärfung von Vorschriften bei den Einleitern von Abwasser in die Kanalisation verwendet wurde (Grenzwert-Überschreitungen bei Schadstoffen aus Gewerbe und Industrieeinleitungen). Weiter diente er als analytisch-qualitativer Indikator zur Beurteilung des Fortschritts in der Begrenzung von Schadstoffen in der Umwelt ganz allgemein. Es wurde festgehalten, dass Klärschlamm auch in Zukunft als "Schadstoff-Indikator" (spezifische Maßnahmen bei den Ursachen von umweltproblematischen Schadstoffanreicherungen im zu beseitigenden Klärschlamm) dienen soll (UVEK, 2002).

Am 14. September 2001 wurde in Bern ein Workshop mit allen interessierten Kreisen (Landwirtschaft, Beratung und Forschung, Fachorganisationen, Konsumenten, Großverteiler etc.) durchgeführt. Die Teilnehmenden wurden über die Ergebnisse der Risikoanalyse informiert. Anschließend wurde die Haltung der Bundesämter vorgestellt und in verschiedenen Arbeitsgruppen diskutiert. Die Voten waren zum Teil emotionsgeladen und verschiedene Personen verliehen ihrem Unmut über die Situation Ausdruck. Trotzdem zeigte der Workshop eine starke zustimmende Mehrheit zu den Vorschlägen der Bundesämter.

Ein wichtiger Treiber beim Ausstieg aus der landwirtschaftlichen Klärschlammverwertung war letztlich vor allem die Situation auf dem Markt und die absehbare Machbarkeit der Entsorgung von Klärschlamm auch bei Verzicht auf die landwirtschaftliche Klärschlammverwertung. Die Situation auf dem Markt um 2000 kann wie folgt beschrieben werden (CHARDONNENS und CANDINAS, 2002): (i) Nachdem die Bio-Verordnung seit ihrem Inkrafttreten am 1. Januar 1997 Klärschlamm als Dünger in der BioProduktion nicht mehr zuließ, fühlten sich die übrigen Mitspieler auf dem Nahrungsmittelmarkt zunehmend unter Zugzwang. (ii) Im Juni 2001 hatten der Schweizerische Bauernverband, der Verband Schweizerischer Milchproduzenten und weitere landwirtschaftliche Organisationen den Verzicht auf die Klärschlammverwendung in der Landwirtschaft empfohlen. (iii) Im gleichen Zeitraum gaben die in der Schweiz dominierenden Großverteiler Migros und Coop bekannt, dass die Düngung mit Klärschlamm für bestimmte Label nicht mehr zugelassen sei. Bei anderen Labelprodukten liefen ähnliche Diskus- 
sionen. Aufgrund des hohen Anteils der Labelproduktion in der schweizerischen Landwirtschaft wurde die landwirtschaftliche Klärschlammverwertung faktisch unmöglich.

Die Entsorgung von Klärschlamm ohne landwirtschaftliche Klärschlammverwertung erschien machbar, weil die Behörden der Kantone angesichts des über die Jahre stark schwankenden Absatzes von Klärschlamm in der Landwirtschaft früh den Aufbau eines zweiten Entsorgungsweges durch Verbrennung veranlasst hatten. Die Situation präsentierte sich im Jahr 2000 wie folgt: Jährlich fielen in den rund 900 Schweizer Kläranlagen etwa 4 Mio t flüssiger Klärschlamm entsprechend einer TS-Menge von rund $200000 \mathrm{t}$ an. Etwa $40 \%$ dieser Menge wurden in der Landwirtschaft verwertet. Im Jahr 2002 waren es, vermutlich aufgrund des vorgesehenen Ausstiegs aus der landwirtschaftlichen Klärschlammverwertung, nur noch rund $20 \%$. Insgesamt stand eine Trocknungskapazität von rund $47000 \mathrm{t}$ TS, eine Verbrennungskapazität für flüssigen Schlamm von $102000 \mathrm{t}$ TS und für getrockneten Schlamm von rund 60000 t TS zur Verfügung. Nur $2 \%$ des Klärschlammanfalls wurden mangels Verbrennungskapazitäten auf Deponien entsorgt. Bei einem Anfall von $202000 \mathrm{t}$ TS Klärschlamm fehlte somit eine Verbrennungskapazität von $40000 \mathrm{t}$ TS, um sämtlichen Klärschlamm in der Schweiz zu entsorgen. Für diese Menge war der Export in ausländische Anlagen (z.B. Braunkohlekraftwerke) geplant, bis die erforderliche Entsorgungsinfrastruktur bereitstehen würde. Im Jahr 2003 stand eine Verbrennungskapazität für Klärschlamm von 166000 t TS zur Verfügung und für das Jahr 2006 wurde eine Verbrennungskapazität von rund $206000 \mathrm{t}$ bei einem Anfall von 204000 t TS Klärschlamm prognostiziert (LAUBE und VONPLON, 2004). Im Jahr 2006 präsentierte sich die Situation wie folgt (mündliche Mitteilung K. SCHENK, Bundesamt für Umwelt, Bern): Von den insgesamt rund 205000 t TS Klärschlamm wurden etwa $180000 \mathrm{t}$ TS verbrannt. Je ein ca. Viertel davon gelangte in Kehrichtverbrennungsanlagen und Zementwerke sowie etwa die Hälfte in Schlammverbrennungsanlagen (SVA) bzw. industrielle Feuerungen. Obwohl genügend Verbrennungskapazitäten vorhanden waren, wurden noch etwa $0,3 \%$ des Schlamms auf Deponien entsorgt und ca. $1 \%$ exportiert. Diese Zahlen zeigen, dass die Schweiz wie geplant die autonome Entsorgung von Klärschlamm ab dem Jahr 2006 erreicht hat, ohne sich mit umstrittenen Lösungen wie der Deponierung behelfen zu müssen. $\mathrm{Zu}$ bemängeln wäre lediglich die ungleiche geografische Verteilung der Verbrennungskapazitäten. Größere Mengen von Klärschlamm müssen deshalb in entwässerter oder getrockneter Form zu den Anlagen transportiert werden.

\subsection{Alternativen zur Rezyklierung von Nährstoffen}

Trotz allem wurde der Ausstieg aus der landwirtschaftlichen Klärschlammverwertung als Rückschlag im Bemühen um Schließung von Stoffkreisläufen und Schonung der Ressourcen wahrgenommen. So wurde befürchtet, dass mit einem Verzicht auf die landwirtschaftliche Klärschlammverwertung auch der Anreiz wegfällt, die Schadstoffbelastung des Abwassers gering zu halten. Es wurde postuliert, dass die vorsorgliche Schadstoffminimierung in den Konsum- und Investitionsgütern deshalb auch weiterhin eine klare Priorität der Umweltpolitik bleiben soll (CHARDONNENS und CANDINAS, 2002). Inwieweit dies gelingt, könnten Zeitreihen von Klärschlammanalysen zeigen. Momentan werden solche Monitoringprogramme von den Bundesbehörden jedoch nicht finanziert.

Die neue Situation bot jedoch auch die Chance, neue Möglichkeiten zur Rezyklierung von Nährstoffen (insbesondere von Phosphor) aus dem Abwasser bzw. aus dem Klärschlamm zu evaluieren (CHARDONNENS und CANDINAS, 2002) und gleichzeitig dessen Befrachtung mit einer Vielzahl von Schadstoffen $\mathrm{zu}$ vermeiden. Eine der Möglichkeiten ist die Rückgewinnung von $\mathrm{P}$ aus Aschen der Monoverbrennung von Klärschlamm. Ein Projekt des Instituts für Pflanzenwissenschaften der ETH Zürich untersucht den Effekt eines solchen P-Düngers nach Extraktion der Schwermetalle (Verfahren der ASH DEC Umwelt AG, Österreich) hinsichtlich Pflanzenernährung, Bodenfruchtbarkeit und Wirtschaftlichkeit (http://www. pe.ipw.agrl.ethz.ch/research/projects). Eine andere Strategie basiert auf der Abtrennung der Wertstoffe vor der Einleitung in die Kanalisation. Das Projekt Novaquatis der Eawag untersucht die Machbarkeit der getrennten
Sammlung von Urin. Dieses Projekt zeigte, dass die NoMix-Technologie eine gute Alternative zur heutigen Nährstoffelimination werden könnte. Voraussetzung ist, dass eines der beiden grundlegenden Probleme gelöst wird: Entweder findet man eine attraktive, breit einsetzbare und kostengünstige Lösung für den Transport des Urins oder man entwickelt dezentrale Verfahren, die stabil und wirtschaftlich sind, um den Urin lokal aufzubereiten (LARSEN und LIENERT, 2007).

Abgesehen von diesen beiden Projekten sind momentan in der Schweiz keine Vorhaben zur Rezyklierung von Nährstoffen aus dem Abwasser oder aus dem Klärschlamm im Gange. Das Verbot der landwirtschaftlichen Klärschlammverwertung hat demnach nur in begrenztem Ausmaß zusätzliche Aktivitäten im Bemühen um Alternativen zur stofflichen Verwertung der im Klärschlamm enthaltenen Nährstoffe ausgelöst.

\section{SCHLUSSFOLGERUNGEN}

Die Resultate der in Kapitel 2 vorgestellten Untersuchungen zeigen, dass Klärschlamm eine Vielzahl von organischen Schadstoffen enthält. Solche Erkenntnisse dürften teilweise zum Ausstieg aus der landwirtschaftlichen Klärschlammverwertung beigetragen haben. Begründet wurde dieser Schritt denn auch mit gesundheitlichen und ökotoxikologischen Risiken. Weitere Treiber waren das Marktrisiko sowie die relativ gute Umsetzbarkeit der Verbrennung von Klärschlamm. Inwieweit Klärschlamm weiterhin als Beurteilungsgrundlage für die Überprüfung der Einleitung von Problemstoffen ins Abwasser und als Instrument zur Beurteilung des Fortschritts bei der Begrenzung von Schadstoffen in der Umwelt ganz allgemein verwendet wird, ist derzeit offen. $\mathrm{Ob}$ nach dem Ausbringungsverbot die direkte Verwertung von Klärschlamm auf die Böden durch umweltfreundlichere Alternativen zur Wiederverwertung der Nährstoffe abgelöst wird, lässt sich ebenfalls noch nicht abschließend beurteilen. Positive Auswirkungen des Klärschlammverbots in der Landwirtschaft sind die Entlastung der landwirtschaftlichen Böden von relevanten Schadstofffrachten und die allgemein erhöhte Sensibilität bezüglich Schadstoffe bei Recyclingdüngern. 


\section{LITERATUR}

BERSET, J.D., HOLZER, R. (1999) Quantitative determination of polycyclic aromatic hydrocarbons, polychlorinated biphenyls and organochlorine pesticides in sewage sludges using supercritical fluid extraction and mass spectrometric detection. J Chromatogr A 852: 545-558.

BERSET, J.D., HOLZER, R. (2001) Determination of phthalates in crude extracts of sewage sludges by high-resolution capillary gas chromathography with mass spectrometric detection. J AOAC Intern 84 (2): 383-391.

BRÄNDLI, R.C., BUCHELI, T.D., KUPPER, T., FURRER, R., STAHEL, W., STADELMANN, F.X., TARRADELLAS, J. (2007a) Organic pollutants in Swiss compost and digestate. 1. Polychlorinated biphenyls, polycyclic aromatic hydrocarbons and molecular markers, determinant processes and source apportionment. J Environ Monit 9:465-464.

BRÄNDLI, R.C., KUPPER, T., BUCHELI, T.D., ZENNEGG, M., HUBER, S., ORTELLI, D., MÜLLER, J., SCHAFFNER, C., IOZZA, S., SCHMID, P., BERGER, U., EDDER, P., OEHME, M., STADELMANN, F.X., TARRADELLAS, J. (2007b) Organic pollutants in Swiss compost and digestate; 2. Polychlorinated dibenzo-p-dioxins, and -furans, dioxin-like polychlorinated biphenyls, brominated flame retardants, perfluorinated alkyl substances, pesticides and other compounds. J Environ Monit 9:465-472.

CHARDONNENS, M., CANDINAS, A. (2002) Risikobeurteilung der Düngemittel und Auswirkungen für die Entsorgungswirtschaft - Anmerkungen aus der Schweiz In: KLAGES, S., ROTH, U. (eds.) Landwirtschaftliche Verwertung von Klärschlamm, Gülle und andern Düngern unter Berücksichtigung des Umwelt- und Verbraucherschutzes. BMU / BMVEL, Wissenschaftliche Anhörung, 25.-26.Oktober 2001 in Bonn. KTBLSchrift 404. Darmstadt: Kuratorium für Technik und Bauwesen in der Landwirtschaft e.V. (KTBL). pp 255-264.

HERTER, U., KUPPER, T., KÜLLING, D. (2003) Risikoabschätzung zur landwirtschaftlichen Abfalldüngerverwertung. Schriftenreihe der FAL 48. Eidg. Forschungsanstalt für Agrarökologie und Landbau, Zürich-Reckenholz.

KUPPER, T., BERSET, J.D., ETTER-HOLZER, R., FURRER, R., TARRADELLAS, J. (2004) Concentrations and specific loads of polycyclic musks in sewage sludge originating from a monitoring Network in Switzerland. Chemosphere 54 (8): 1111-1120.

KUPPER, T., DE ALENCASTRO, L.F., GATSIGAZI, R., FURRER, R., GRANDJEAN, D., TARRADELLAS, J. (2007) Concentrations and specific loads of brominated flame retardants in sewage sludge. Chemosphere: in press. doi:10.1016/j.chemosphere.2007.10.019.
KUPPER, T., PLAGELLAT, C., BRÄNDLI, R.C., DE ALENCASTRO, L.F., GRANDJEAN, D., TARRADELLAS, J. (2006) Fate and removal of polycyclic musks, UV filters and biocides during wastewater treatment. Water Res 40 (14): 2603-2612.

LARSEN, T.A., LIENERT, J. (2007) Novaquatis Abschlussbericht. NoMix - Neue Wege in der Siedlungswasserwirtschaft. Eawag, 860o Dübendorf, Schweiz.

LAUBE, A., VONPLON, A. (2004) Klärschlammentsorgung in der Schweiz Mengen- und Kapazitätserhebung. Umweltmaterialien Nr. 181. Abfall. Bundesamt für Umwelt, Wald und Landschaft BUWAL.

PLAGELLAT, C., KUPPER, T., DE ALENCASTRO, L.F., GRANDJEAN, D., TARRADELLAS, J. (2004) Biocides in sewage sludge: quantitative determination in some Swiss wastewater treatment plants. Bull Environ Contam Toxicol 73 (5): 794-801.

PLAGELLAT, C., KUPPER, T., FURRER, R., DE ALENCASTRO, L.F., GRANDJEAN, D., TARRADELLAS, J. (2006) Concentrations and specific loads of UV filters in sewage sludge originating from a monitoring network in Switzerland. Chemosphere 62 (6): 915-925.

SCHWARZENBACH, R.P., ESCHER, B.I., FENNER, K., HOFSTETTER, T.B., JOHNSON, A.C., VON GUNTEN, U., WEHRLI, B. (2006) The challenge of micropollutants in aquatic systems. Science 313: 1072-1077.

SELLSTRÖM, U., DE WIT, A.A., LUNDGREN, N., TYSKLIND, M. (2005) Effect of sewage-sludge application on concentrations of higher-brominated diphenyl ethers in soils and earthworms. Environ Sci Technol 39 (23): 9064-9070.

UVEK (2002) Erläuterungen zur Änderung der Klärschlammvorschriften in der Stoffverordnung und in weiteren Bundesverordnungen mit Klärschlammvorschriften. Eidgenössisches Departement für Umwelt, Verkehr, Energie und Kommunikation, Bern.

WETTSTEIN, F. (2004) Auftreten und Verhalten von Nonylphenoxyessigsäure und weiteren Nonylphenolverbindungen in der Abwasserreinigung. Diss. ETH Nr. 15315. ETH Zürich.

Anschrift des Verfassers: Thomas KUPPER, Schweizerische Hochschule für Landwirtschaft, SHL, Länggasse $\mathrm{CH}-3052$ Zollikofen, Schweiz 